\title{
Robust Autocalibration for a Surveillance Camera Network
}

\author{
Jingchen Liu, Robert T. Collins, and Yanxi Liu \\ The Pennsylvania State University \\ University Park, PA 16802, USA \\ \{jingchen, rcollins, yanxi\}@cse.psu.edu
}

\begin{abstract}
We propose a novel approach for multi-camera autocalibration by observing multiview surveillance video of pedestrians walking through the scene. Unlike existing methods, we do NOT require tracking or explicit correspondences of the same person across time/views. Instead, we take noisy foreground blobs as the only input and rely on a joint optimization framework with robust statistics to achieve accurate calibration under challenging scenarios. First, each individual camera is roughly calibrated into its local World Coordinate System (IWCS) based on analysis of relative $3 D$ pedestrian height distribution. Then, all lWCSs are iteratively registered with respect to a shared global World Coordinate System ( $g$ WCS) by incorporating robust matching with a partial Direct Linear Transform ( $p D L T)$. As demonstrated by extensive evaluation, our algorithm achieves satisfactory results in various camera settings with up to moderate crowd densities with a large proportion of foreground outliers.
\end{abstract}

\section{Introduction}

The main goal of surveillance camera calibration is to find the mapping relating objects in the 3D scene to their projections in the 2D image plane[9]. This helps to infer object locations as well as scales and orientations, and allows for more accurate object detection and tracking. For example, sampling-based pedestrian detection [1, 4] yields better performance when hypotheses are generated in 3D and then projected into one or more image views. Pedestrian/face/object detection based on sliding windows can also benefit from calibration, since the search over orientation and scale can be constrained to a small range, reducing false positives [13].

In this paper, we present an automated calibration method that enables smart sampling of object size and orientation in all views given either a $2 \mathrm{D}$ location in one view or 3D location in the scene. The method works directly on noisy foreground observations collected by the surveillance

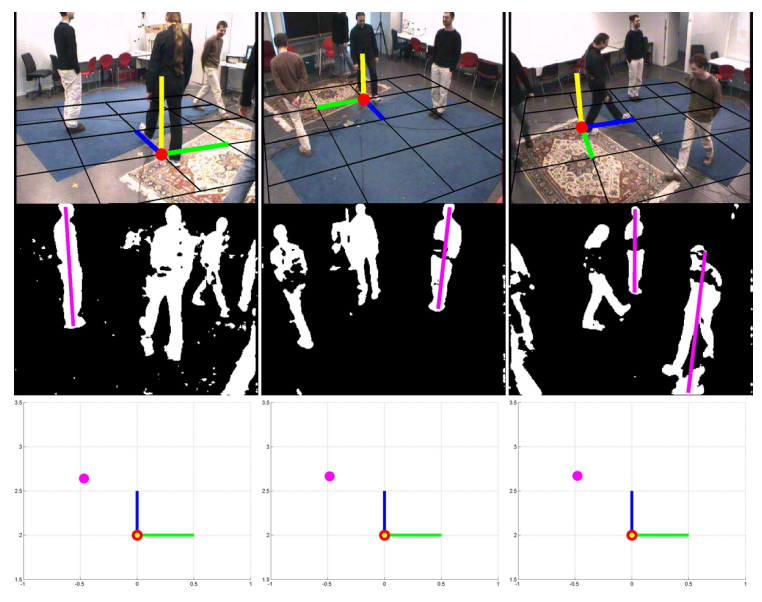

Figure 1. Example frames for calibration: (top) original frame overlayed with calibration results; (middle) noisy foreground masks with major axes of inlier blobs; (bottom) registered topdown view with the same blob

system, without any further information such as scene geometry or tracklets from tracking.

Most existing work on unsupervised surveillance (pedestrian-based) camera calibration focuses on the singleview case, $([13,7,15,12,8])$ and requires clean pedestrian detections as well as explicit correspondences of the same person at different locations in the scene. For example, [12] proposes to detect leg-crossings for more accurate pedestrian height estimation; [13] requires the extraction of multiple control points on the contour of the pedestrian. [3, 6] and [14] adopt similar ideas and use a walking human to calibrate a camera network. In all the above work, the correspondence of the same person, if not manually labeled, is obtained either by tracking, or under the assumption that there is only one person in the view.

In some cases however, it can be very difficult to accurately detect pedestrians prior to calibration, let alone track them robustly through the scene. [11] takes noisy foreground blobs as input and achieves camera calibration based on the analysis of pedestrian height distribution, with no correspondence information needed. However the estima- 
tion of focal length may not be very accurate, and it only applies to single views. To the best of our knowledge, the automatic extraction of cross-view correspondences in noisy environments has not been addressed in the above work.

It is known that once clean correspondences (of points, planes, objects in 2D/3D) are given, calibration is a wellsolved problem, e.g., using bundle adjustment[16]. The main contribution of this work is to propose a novel framework for unsupervised surveillance system calibration that efficiently prunes outliers and estimates the calibration based on a subset of inlier foreground correspondences discovered through applying a series of robust statistics.

We address four major challenges that are commonly encountered but not fully considered in the existing literature on surveillance-based calibration: (1) moderately crowded scenes; (2)a large proportion of outliers from foreground extraction; (3) large noise (variance) in foreground detections; (4) no correspondence information across frames/views. Similar to most surveillance work, we assume (1) there is one single flat ground-plane and (2) people are almost vertical, standing/walking on the ground plane.

\section{Camera Model and the Coordinate System}

Adopting a simplified CCD camera model with focal length being the only intrinsic parameter, we calibrate each view into its local World Coordinate System (IWCS), where each camera has zero pan angle and is translated from the local origin $O_{L}$ by one unit along the $\mathrm{Z}$-axis (thus the relative scale of the coordinate system is proportional to the camera height above the ground). Camera orientation is modeled by a tilt angle $\theta$ around the $\mathrm{X}$-axis $(\theta \in(\pi / 2, \pi)$ for a downward looking camera) and a roll angle $\rho$ around the Z-axis. The 3D-to-2D projection matrix is thus defined by:

$$
P_{L}=\left[\begin{array}{lll}
f & & \\
& f & \\
& & 1
\end{array}\right] R_{Z}(\rho) R_{X}(\theta)\left[\begin{array}{lll|c}
1 & & & 0 \\
& 1 & & 0 \\
& & 1 & -1
\end{array}\right],
$$

where, e.g., $R_{X}(\theta)$ is a $3 \mathrm{D}$ rotation around the $\mathrm{X}$-axis by angle $\theta$.

It has been shown [10] that the local extrinsic parameters $(\rho, \theta)$ can be estimated given the vertical vanishing point $v_{0}=\left(v_{x}, v_{y}, 1\right)^{T}$ together with the focal length $f$ as:

$$
\begin{aligned}
v_{x} x & +v_{y} y+f^{2}=0 \\
\rho & =\operatorname{atan}\left(-v_{x} / v_{y}\right) \\
\theta & =\operatorname{atan} 2\left(\sqrt{v_{x}^{2}+v_{y}^{2}},-f\right),
\end{aligned}
$$

where Eqn. 2 is the equation of the horizon.

To relate all IWCSs, we choose a global World Coordinate System (gWCS) that is aligned to the ground plane so that each IWCS can be registered with the gWCS by a $2 \mathrm{D}$

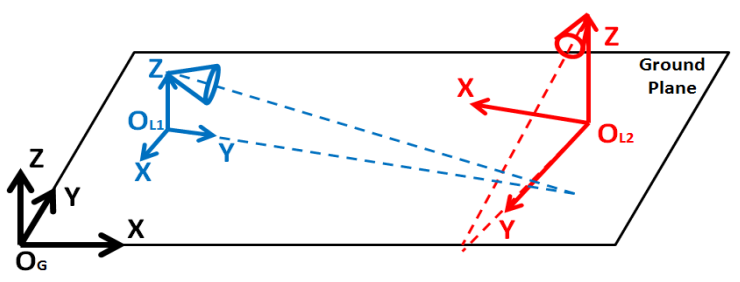

Figure 2. Illustration of the global WCS (black) and the local WCSs (blue and red), where the local Y-axis is coplanar with the camera optical axis and all XY-planes lie within the ground-plane.

translation and rotation within the ground plane (XY-plane), as well as a relative scaling (proportional to the individual camera height), as illustrated in Fig. 2. The final projection matrix of a camera is defined as

$$
P=P_{L} \cdot P_{G}
$$

where $P_{G}$ denotes the ground-plane alignment transformation,

$$
P_{G}=\left[\begin{array}{ccccc}
s & & & \\
& s & & \\
& & s & \\
& & & 1
\end{array}\right]\left[\begin{array}{cccc}
\cos \alpha & -\sin \alpha & T_{x} \\
\sin \alpha & \cos \alpha & & T_{y} \\
& & 1 & 0 \\
& & & 1
\end{array}\right]
$$

\section{Camera Calibration}

Our algorithm works on videos captured by multiple cameras with overlapping views. The original frames are preprocessed to generate foreground blobs. From these noisy blobs in each single view, we first estimate the vertical vanishing point and the maximum-likelihood focal length $f$ that recovers $3 \mathrm{D}$ blob heights resembling the real world distribution of human heights using [11], thus estimating the individual camera calibration matrix $P_{L}^{(k)}$ that relates the coordinate system of camera $k$ to its local WCS. We then iteratively and sequentially modify the global registration $\left(P_{G}\right)$ of each camera. The iteration usually converges in a few rounds (2 to 3 ). During each iteration, we minimize the re-projection error between the $2 \mathrm{D}$ blobs in the current view and the joint set of global-world 3D blobs maintained by all cameras, where the correspondence information is implicitly encoded in a robust-statistic error metric. We then efficiently solve for a final estimate of $P_{G}$ via partial Direct Linear Transform (pDLT) in a reduced solution space. The workflow of the algorithm is summarized in Alg. 1.

\subsection{Framework}

Following adaptive background subtraction on the input video sequences, we merge connected foreground pixels to form foreground blobs. We then fit an ellipse to each blob and represent the blob by the two end points of the major 
axis of the ellipse. Assuming each foreground blob corresponds to a person in the 2D image plane, the two end points approximately represent the pixel locations of the foot and head of the person. Denote by $b_{n}^{(k)}$ the $n$th $2 \mathrm{D}$ blob extracted from the $k$ th view and $B_{n}^{k}$ the corresponding 3D blob in the gWCS. These 2D and 3D blobs can be represented in homogeneous coordinates as:

$$
b_{n}^{(k)}=\left[\begin{array}{cc}
x_{f} & x_{h} \\
y_{f} & y_{h} \\
1 & 1
\end{array}\right] \quad B_{n}^{(k)}=\left[\begin{array}{cc}
X & X \\
Y & Y \\
0 & H \\
1 & 1
\end{array}\right],
$$

where $x_{f}, y_{f}, x_{h}, y_{h}$ are pixel locations of the foot and head, and $X, Y, H$ indicate the pedestrian's location (in the ground plane) and height respectively. The projective matrix $P^{(k)}$ (Eqn. 6) projects a 3D blob into 2D: $b_{n}^{(k)} \sim$ $P^{(k)} \cdot B_{n}^{(k)}$. Assuming upright pedestrians walking in the ground-plane, the degree of freedom (DoF) of $\left\{B_{n}^{(k)}\right\}$ is 3. Thus $B_{n}^{(k)}$ can be linearly solved given $P_{3 \times 4}^{(k)}$ and $b_{n}^{(k)}$. Specifically, let

$$
\begin{gathered}
M=\left[\begin{array}{ccc}
x_{f} P_{31}-P_{11} & x_{f} P_{32}-P_{12} & 0 \\
y_{f} P_{31}-P_{21} & y_{f} P_{32}-P_{22} & 0 \\
x_{h} P_{31}-P 11 & x_{h} P_{32}-P_{12} & x_{h} P_{33}-P_{13} \\
y_{h} P_{31}-P_{21} & y_{h} P_{32}-P_{22} & y_{h} P_{33}-P_{23}
\end{array}\right], \\
t=\left[\begin{array}{c}
P_{14}-x_{f} P_{34} \\
P_{24}-y_{f} P_{34} \\
P_{14}-x_{h} P_{34} \\
P_{24}-x_{h} P_{34}
\end{array}\right],
\end{gathered}
$$

it can be proven that $[X, Y, H]^{T}=\left(M^{T} M\right)^{-1} M^{T} t$. We denote such a backward projection from $2 \mathrm{D}$ to $3 \mathrm{D}$ as: $B_{n}^{(k)}=\left\{P^{(k)}\right\}^{-1} b_{n}^{(k)}$. Note that the $\{\cdot\}^{-1}$ operator here does not refer to the conventional matrix inversion.

We formulate the multi-camera calibration as a joint energy minimization problem. Among various choices of the cost function for multi-camera calibration, we select the widely used mean-squared image re-projection error as our optimization goal, which can also be interpreted as maximum likelihood estimation under an assumption of Gaussian noise[5]. The re-projection from view $j$ to view $k$ can be expressed as:

$$
\begin{aligned}
b_{n}{ }^{(k \mid j)} & \sim P^{(k)}\left\{P^{(j)}\right\}^{-1} b_{n}^{(j)} \\
& \sim P^{(k)} B_{n}^{(j)}
\end{aligned}
$$

where a $2 \mathrm{D}$ blob in view $j$ is first back-projected into the gWCS with operator $\left\{P^{(j)}\right\}^{-1}$ and then projected into view $k$ under $P^{(k)}$. The overall re-projection error is defined by

$$
\begin{aligned}
\varepsilon & =\sum_{k} \varepsilon^{(k)}=\sum_{k} \sum_{j} e\left(b^{(k)}, b^{(k \mid j)}\right) \\
& =\sum_{k} e\left(b^{k}, P^{(k)} B\right)
\end{aligned}
$$

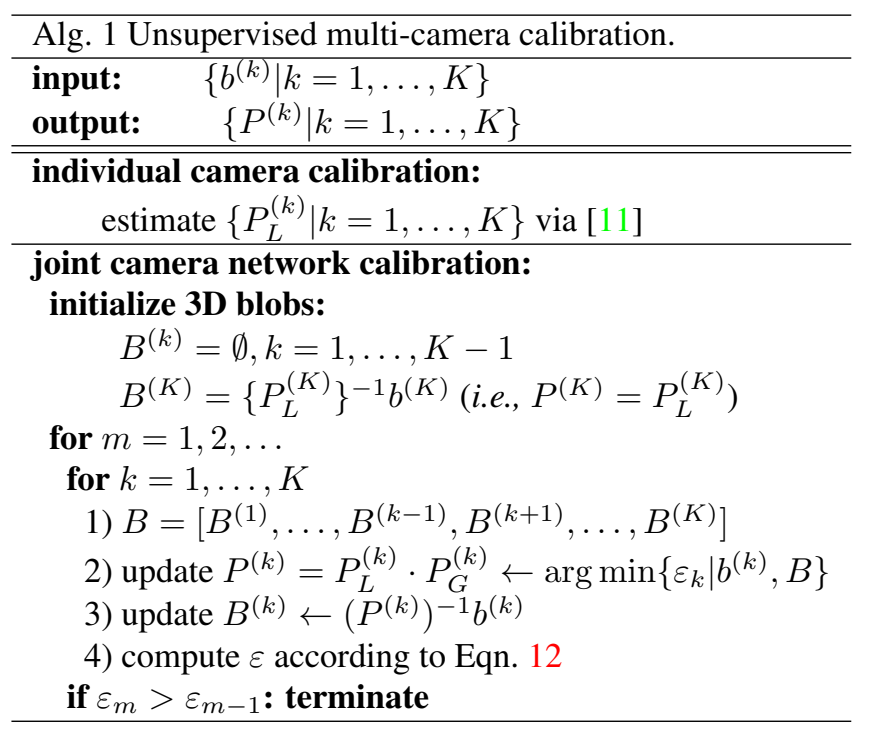

where $B=\left(B^{(1)}, \ldots, B^{(K)}\right)$ is the set of 3 D blobs contributed by all views. Note that the self back-projection always has $b_{n}^{k \mid k}=b_{n}^{k}$. Hence $e\left(b^{k}, P^{(k)} B^{(k)}\right)$ is always 0 . $e($,$) is a robust matching error metric, defined in Sec. 3.3$ that measures the compatibility between the set of foreground blobs $b^{k}$ observed in view $k$ and the set of reprojected blobs from 3D $(B)$, as contributed by all views, where no correspondence information between $b^{k}$ and $B$ is given.

Minimizing the above cost function directly is intractable. However, as can be seen from Eqn. 12, we can iteratively optimize the projection matrix for each view. To initiate the IWCS-gWCS matching, we first align the gWCS with one of the IWCSs (here we pick the last view indexed by $K$ ) to obtain an initial set of 3D blobs. Then we sequentially calibrate each camera $k$ by (1) inferring the correspondences between $b^{(k)}$ and $B$ under the presence of outliers and noise with a robust matching metric (Sec. 3.3) and (2) optimizing the projective matrix $P^{(k)}$ given the 2D-3D blob correspondences by partial direct linear transformation (Sec. 3.4). Empirically, we observe that our multi-camera calibration usually converges in no more than three iterations, i.e., $m \leq 3$ in Alg. 1 .

\subsection{Individual Camera Calibration to IWCS}

This section explains how we obtain the input matrices $\left\{P_{L}^{(k)}\right\}$ w.r.t. IWCS. As shown in Eqns. 1,3,4, this is equivalent to estimating the focal length $f$ and the vertical vanishing point $v_{0}$. We apply the method of [11] that first uses RANSAC to find the vanishing point and then roughly estimates the focal length based on prior knowledge of the 3D-height distribution of inlier blobs. Note that we do not need to assume a constant person height for calibration, as is often done in algorithms for foot-head homography esti- 

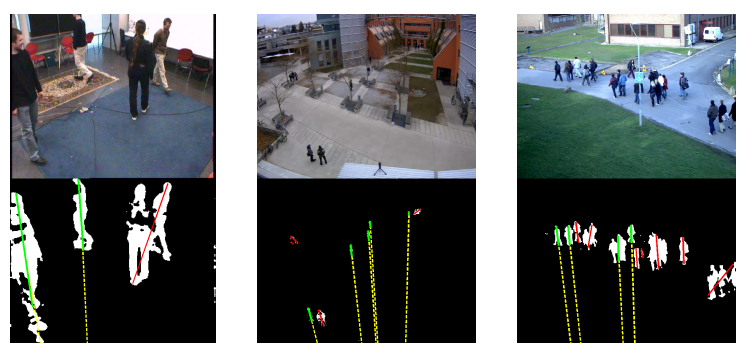

Figure 3. Vanishing point detection under different camera angles, foreground blob sizes, and crowd densities. Green lines indicate the major axes of inlier blobs. Outliers are marked with red lines. Yellow dashed lines indicate the vanishing points.

mation.

The vanishing point estimation is carried out in homogeneous coordinates, and is robust in cases when a vanishing point is close to infinity. Fig. 3 demonstrates a few examples of RANSAC-based vanishing point voting under different camera settings as well as varying foreground sizes and densities. It is worth mentioning that many blobs corresponding to real pedestrians are classified as outliers because of region deformation due to partial detection or being merged with other people, especially in crowded scenes. However our goal is not to detect all pedestrian foreground blobs but to extract enough inlier blobs for the following analysis.

Focal length estimation follows a hypothesize-and-test process. Given a hypothesized focal length $f$, together with the vanishing point $v_{0}$, we can recover the relative 3D heights $H_{i}$ of each inlier blob $b_{i}$ (w.r.t. the camera height $)^{1}$. This process leverages the fact that the distribution of human heights in 3D forms a very strong cluster with $\left|H_{i}-\mu\right| / \mu<\lambda$, where $\mu$ is the average inlier pedestrian height and $\lambda=0.1$ [11]. Different hypotheses are evaluated against a robust log likelihood function defined as:

$$
\mathcal{L}(f)=\frac{1}{\mu^{2}} \sum_{i \in I} \max \left\{\lambda \mu-\left|H_{i}-\mu\right|, 0\right\}^{2},
$$

where $I$ represents the set of RANSAC inliers, and outlier candidates $H_{i}$ that fall out of the height range of the majority of the inlier blobs, e.g., $H_{i}>(1+\lambda) \mu$, are ignored. As we sample the camera field of view (FoV) angle at a resolution of $1^{\circ}$, which is about the state-of-the-art accuracy for pedestrian-based surveillance camera calibration [8], the focal length $f$ that produces the highest likelihood score according to Eqn. 13 is selected as our initial estimate for the multi-view calibration.

\subsection{Robust Distance Metric for Blob Matching}

This section explains the cost function (Eqn. 11) for cross-view IWCS matching. Recall that $b^{(i)}$ denotes the set

\footnotetext{
${ }^{1} \mathrm{~A}$ more efficient method would use the cross-ratio invariance trick [11].
}

of 2D blobs in view $i$ for the entire sequence, $B^{(i)}$ denotes the set of 3D blobs back-projected from view $i$ to gWCS, and $b^{(i \mid j)}$ is the set of re-projected 2D blobs from view $j$ to $i$. The cost function is defined as the sum of the reprojection errors of all pairs of 2D-3D blobs (denoted as set $l$ ) extracted at the same time stamp.

Since the foreground blobs are noisy in the sense of both false alarms and missed detections, the proportion of 'good' pairs (two 'good' blobs extracted from different views corresponding to the same person) is even smaller, especially under crowded scenarios. We thus adopt truncated quadratic[2], which belongs to the robust statistics of truncated least squares, defined as:

$$
e\left(b^{(i)}, b^{(i \mid j)}\right)=\sum_{\left(b_{n_{1}}^{(i)}, B_{n_{2}}^{(j)}\right) \in l} \min \left\{d\left(b_{n_{1}}^{(i)}, b_{n_{2}}^{(i \mid j)}\right)^{2}, \tau^{2}\right\}
$$

where $d($,$) is the 4D Euclidian distance, (x, y)$ coordinates of feet and head, between 2 blobs in pixels, and the error tolerance is set to be $\tau^{2}=\frac{1}{100} W \cdot H$, where $W$ and $H$ are the width and height of the image. We find this setting yields satisfactorily consistent results for video sequences with very different camera settings, as demonstrated in the experiments section. We iteratively use the error tolerance as a threshold to discover 'good' blob correspondences from all possible pairs in $l$ and re-estimate calibration parameters based on these inliers.

\subsection{Multiview Calibration to gWCS by Partial DLT}

This section describes the sequential registration of multiple IWCS in Alg. 1. The goal is to estimate the camera projection matrix $P$ from an initial set of noisy inlier 2D-3D blob correspondences $\left(l_{i n}\right)$ and the results of the single-view calibration $\left(P_{L}\right)$. We propose an iterative process based on a variant of direct linear transform (DLT). The algorithm iteratively estimates the global projection matrix and refines the inlier correspondences $l_{i n}$ and $P_{L}$ once $P_{G}$ has been updated. The overall optimization is summarized in Alg. 2.

To solve the global transformation of the $k$ th view $P^{k}=$ $P_{L}^{k} \cdot P_{G}^{k}$ (Eqn. 6) from a linear system constructed from inlier blob correspondences between the 2D blobs and 3D blobs

$$
[x, y, z]^{T} \sim P_{L}^{k} \cdot P_{G}^{k} \cdot[X, Y, Z, 1]^{T},
$$

a straightforward approach would treat $P$ as a general matrix with $12 \mathrm{DoF}$, and solve it using DLT. However, the DLT solution is known to be overdetermined, as the real perspective matrix only has $11 \mathrm{DoF}$ (up to a scale). More importantly, when the 2D-3D correspondences are noisy, DLT can easily overfit the free-form solution, making it harder to distinguish inlier correspondences from outliers during the iterative process, which would further degrade the estimation accuracy. Therefore, we limit the DoF of the solution space 


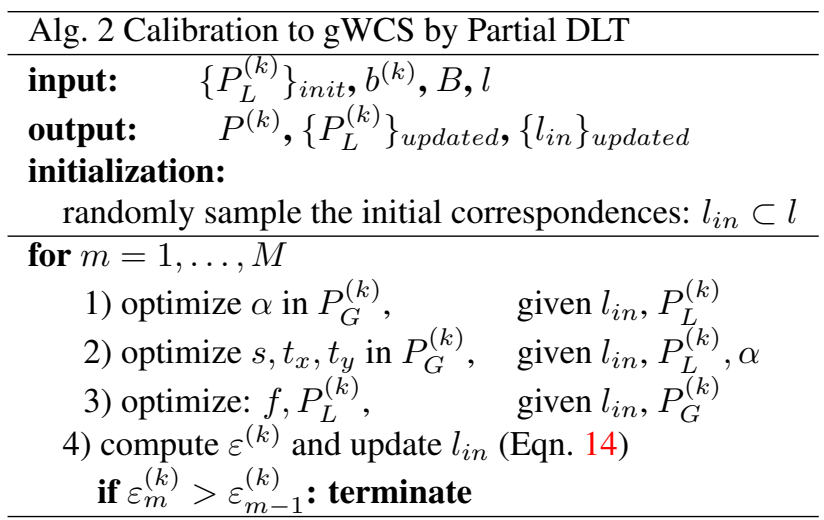

by estimating the five variables in Eqns. 1, 6: $\alpha, s, t_{x}, t_{y}, f$ sequentially, while fixing the vanishing point. The motivation is that (1) we assume the initial estimation for vanishing point $v_{0}$ is accurate enough; (2) by reducing the DoF of the projection matrix, we introduce a partial DLT here to solve for subsets of parameters efficiently without suffering from the 'over-determinant' problem of DLT.

To estimate the ground-plane rotation angle $\alpha$, we fix $f$ and $P_{L}^{k}$ so that $P_{G}^{k}$ has $7 \mathrm{DoF}$ :

$$
P_{G}^{k} \sim P_{G \mid 7}^{k}=\left[\begin{array}{cccc}
m_{11} & m_{12} & & m_{14} \\
m_{21} & m_{22} & & m_{24} \\
& & m_{33} & \\
& & & 1
\end{array}\right] .
$$

$P_{G \mid 7}$ can be directly optimized by linearizing Eqn. 15 similar to the DLT (thus referred to as partial DLT) and the rotation angle can be approximated as $\alpha=\operatorname{atan} 2\left(m_{21}-\right.$ $\left.m_{12}, m_{11}+m_{22}\right)$. We then fix $\alpha$ and $f$, so that $s, t_{x}, t_{y}$ together can be directly linearly solved in the same way (see Appendix for detailed derivation). Although the initial estimation of focal length $f$ may not be very accurate, it constrains the search space to a small region for refining the estimates. To optimize $f$, we fix the current estimatesof the otehr parameters and adopt pDLT again on the linear system of:

$$
\left[\begin{array}{l}
x \\
y \\
z
\end{array}\right] \sim\left[\begin{array}{ccc}
a_{f} & & \\
& a_{f} & \\
& & 1
\end{array}\right] \cdot P_{L}^{k} \cdot P_{G}^{k} \cdot\left[\begin{array}{c}
X \\
Y \\
Z \\
1
\end{array}\right]
$$

with $a_{f}$ being the only parameter. This suggests the optimal focal length should be updated as: $f^{*} \leftarrow a_{f} \cdot f$. We then update $\theta$ in $P_{L}$ with $f^{*}$ according to Eqn. 4 (thus the vanishing point remains the same) and re-evaluate the matching error of Eqn. 14. We only accept $f^{*}$ if it reduces the matching error.

\section{Experiments}

We have conducted extensive evaluation on a synthesized dataset for stress testing, and on four different pub- lic sequences with various camera settings, crowd densities, and background subtraction qualities: (a) indoor 4-person sequence with three views of resolution $288 \times 360$ [1], (b) outdoor campus sequence with two views of $960 \times$ 1280 [14], (c) PETS09 sparse crowd sequence with 4 views $(1,5,6,8)$, where view\# 1 is $576 \times 768$ and the rest are $576 \times 720$, and (d) PETS09 medium density crowd sequence with two views (\#1 and \#2) of resolution $576 \times 768^{2}$.

We also provide quantitative comparison against [11] in terms of focal length estimation, since to our best knowledge, no other existing work estimates surveillance network calibration without correspondence information.

\subsection{Synthesized Dataset for Stress-Testing}

We synthesize a dataset with three cameras of different focal lengths $\left(f_{1}=1000, f_{2}=1200, f_{3}=1000\right)$, viewing angles, ground plane locations, and heights. Multiple pedestrians were synthesized in 3D as feet-head pairs with a height variance of $\pm 10 \%$, and then projected into individual views. In the stress test, we consider three sources of input noise: location noise $\sigma_{0}$, foreground blob recall $r$ and precision $p$ rate. The location noise is produced as zero-mean Gaussian noise added onto the original feet-head pixel locations. The blob-recall and precision indicate false negatives and false positives and are simulated by randomly removing inlier blobs and adding outlier blobs. The default

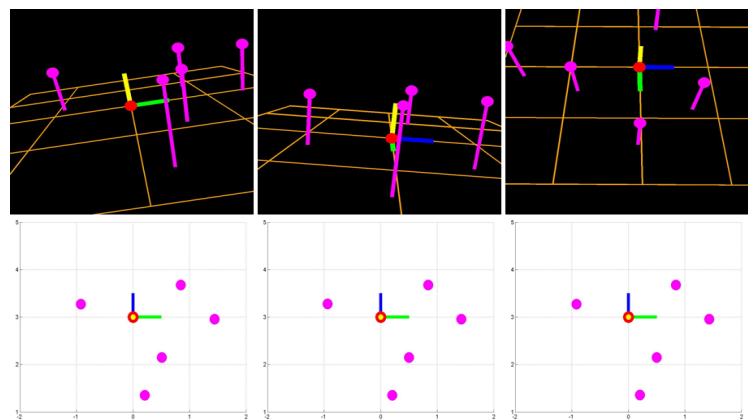

Figure 4. Synthesized 3-camera dataset with calibration results.

stress parameters are set as $\sigma_{0}=5$ (the standard deviation of Gaussian noise in pixels), $r=70 \%$ and $p=70 \%$. We then vary $\sigma_{0}$ from 1 to 10 and $r, p$ from 1 to 0 respectively with the other two parameters fixed at the default level.

Fig. 4 visualizes the calibration results. We also quantitatively evaluate the performance of our algorithm by measuring the average RMSE re-projection error in pixels as well as the average relative focal length estimation error $e=\left|f-f_{G T}\right| / f_{G T}$. The performance of our approach under different stress levels is plotted in Fig. 5.

It can be seen the re-projection error increases with increasing feet-head location noise, but is relatively stable w.r.t. missing inliers and against outliers, which well

\footnotetext{
${ }^{2}$ http://www.cvg.rdg.ac.uk/PETS2009/a.html
} 

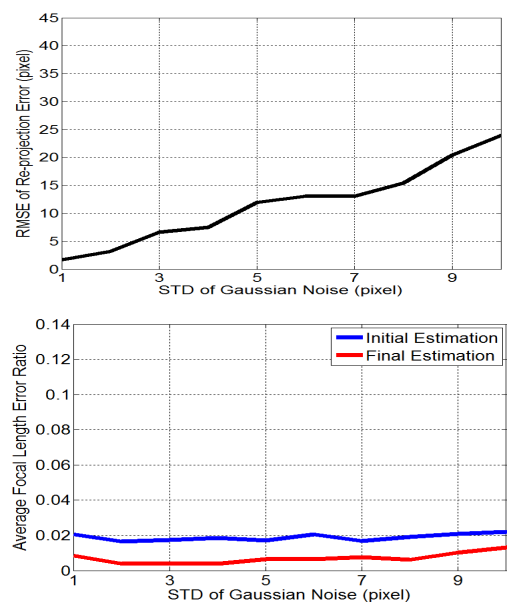

(a)
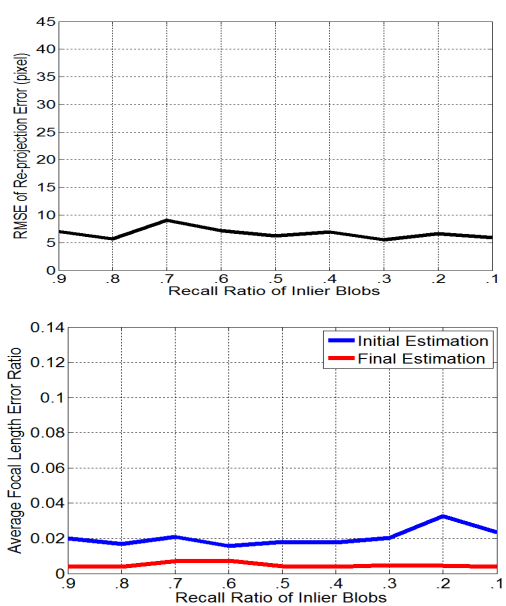

(b)
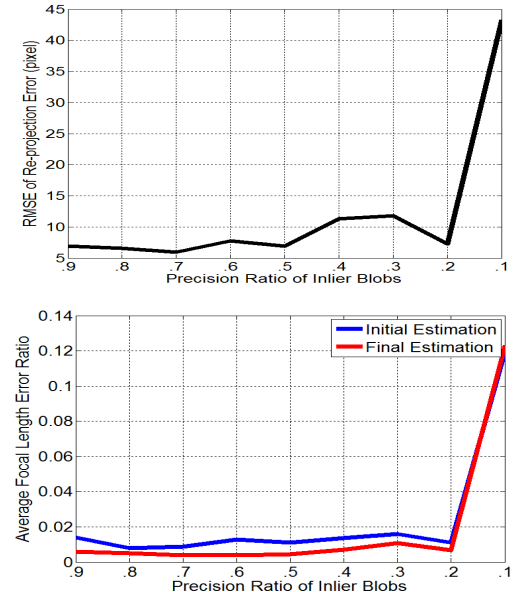

(c)

Figure 5. Performance under different levels of (a) location noise; (b) foreground recall rate; (c) foreground precision rate. Top: average re-projection error. Bottom: focal length error of our approach (red) and [11]

demonstrates the effectiveness of our robust estimation metric. When the precision increases from $p=0.2$ to $p=0.1$ in Fig. 5(bottom), the proportion of outliers increases from $50 \%$ to $90 \%$ and results in a big performance drop. In all other cases, our focal length estimation remains accurate and performs better than a single-view based approach [11].

\subsection{Real Sequences}

As can be seen from the calibration results in Fig. 6, our ground plane estimations are accurate in general (a black grid mask overlaid on the original frame). For quantitative evaluation, we manually labeled corresponding pedestrians across views on 50 frames for each sequence. Fig. 7 compares the algorithm re-projections with the groundtruth labels. The view-specific re-projection error is summarized in Tab. 1, where $E^{i n}$ column indicates the matching error when pedestrians from other views are mapped into the current view, and the $E^{\text {out }}$ column indicates the matching error when pedestrians from the current view are mapped into other views. We show the RMSE in both pixels and in terms of normalized distance as $\mathrm{E}_{r}=\mathrm{E} / \sqrt{W \cdot H}$. Note that some labeling error exists on feet/head positions of the groundtruth labels.

The focal length estimation for sequences with ground truth $f_{G T}$ and relative error $e$ are summarized in Tab. 2. Again, compared with the baseline method using single view calibration, our method achieves better accuracy in most cases. The second view of the campus sequence reported the largest error; however, the cross-view reprojection estimation and groundplane estimation are still acceptable. This can be explained as a case when inaccurate focal length estimation cancels out the inaccurate van- ishing point estimation, resulting in a small re-projection error, which is the primary goal for surveillance calibration.

\begin{tabular}{|c|c||c|c|c|c|}
\hline & View & $E^{\text {in }}$ & $E^{\text {out }}$ & $E_{r}^{\text {in }}$ & $E_{r}^{\text {out }}$ \\
\hline \hline \multirow{3}{*}{ indoor } & 1 & 13 & 16 & $4.0 \%$ & $5.0 \%$ \\
& 2 & 23 & 12 & $7.1 \%$ & $3.6 \%$ \\
& 3 & 14 & 22 & $4.3 \%$ & $6.8 \%$ \\
\hline campus & 1 & 36 & 24 & $3.3 \%$ & $2.2 \%$ \\
& 2 & 24 & 36 & $2.2 \%$ & $3.3 \%$ \\
\hline & 1 & 10 & 34 & $1.6 \%$ & $5.1 \%$ \\
PETS09 & 2 & 29 & 12 & $4.7 \%$ & $2.0 \%$ \\
(sparse) & 3 & 13 & 11 & $2.0 \%$ & $1.8 \%$ \\
& 4 & 16 & 12 & $2.5 \%$ & $1.8 \%$ \\
\hline PETS09 & 1 & 18 & 8 & $2.6 \%$ & $1.2 \%$ \\
(dense) & 2 & 8 & 18 & $1.2 \%$ & $2.6 \%$ \\
\hline
\end{tabular}

Table 1. View specific re-projection error on real sequences.

\begin{tabular}{|c|c||c|c|c|c|c|}
\hline & View & $f_{G T}$ & $f_{\text {base }}$ & $e_{\text {base }}$ & $f$ & $e$ \\
\hline \hline Campus & 1 & 1057 & 1044 & $1 \%$ & 1034 & $2 \%$ \\
& 2 & 1198 & 1545 & $29 \%$ & 1427 & $\mathbf{1 9} \%$ \\
\hline & 1 & 1170 & 1084 & $7 \%$ & 1218 & $\mathbf{4} \%$ \\
PETS09 & 5 & 830 & 828 & $.2 \%$ & 828 & $.2 \%$ \\
(sparse) & 6 & 877 & 891 & $2 \%$ & 869 & $\mathbf{1} \%$ \\
& 8 & 737 & 772 & $5 \%$ & 772 & $5 \%$ \\
\hline PETS09 & 1 & 1170 & 950 & $19 \%$ & 1067 & $\mathbf{9} \%$ \\
(dense) & 2 & 659 & 624 & $5 \%$ & 634 & $\mathbf{4} \%$ \\
\hline
\end{tabular}

Table 2. Focal length estimation, where $f_{G T}$ is the groundtruth focal length, $f_{\text {base }}$ and $f$ are estimates computed by the baseline([11]) and our method, respectively, and $e_{b a s e}$ and $e$ are relative errors w.r.t. the groundtruth. Our method outperforms the baseline in most cases (shown in bold). 


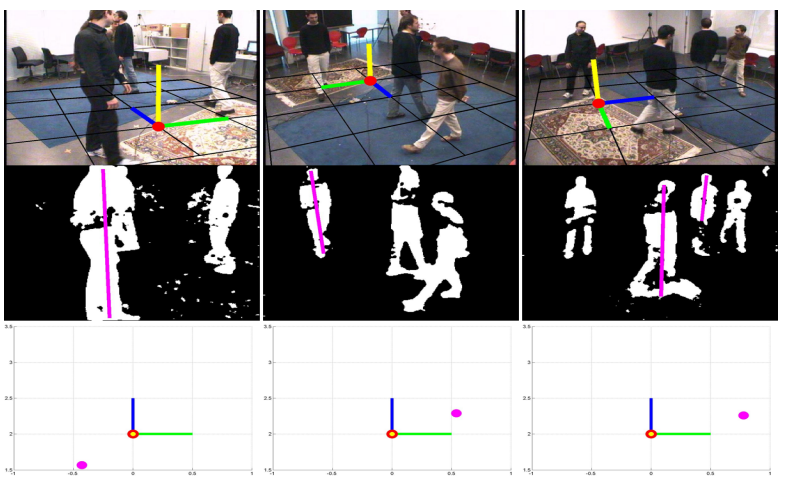

(a)

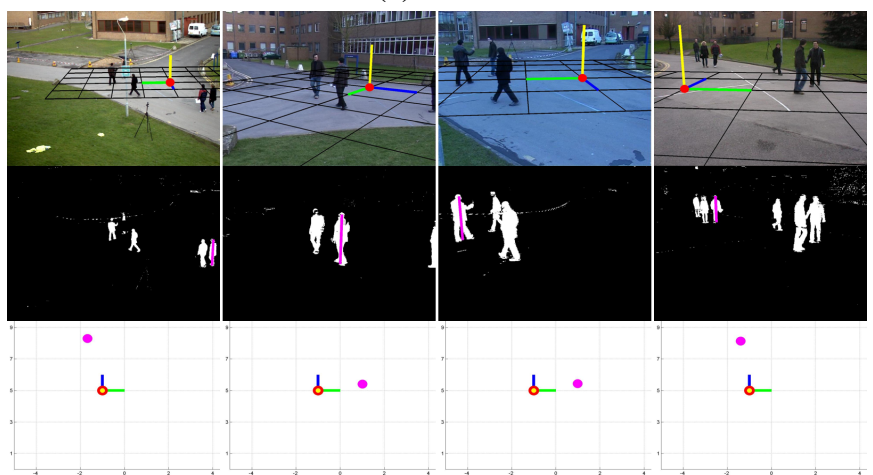

(a)

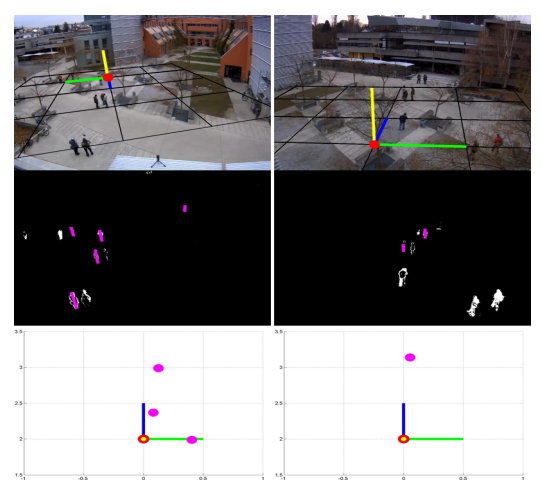

(b)

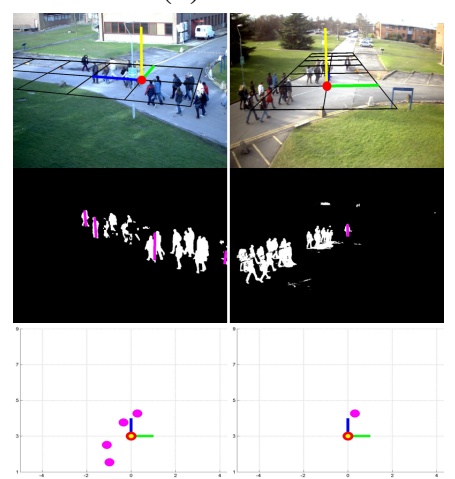

(b)

Figure 6. Example of calibration results for: (a) indoor 4-people sequence; (b) outdoor campus sequence; (c) PETS09 sparse; (d) PETS09 dense. For each sequence: Top: original frame overlayed with calibration results. Middle: noisy foreground masks overlayed with major axes of inlier blobs. Bottom: registered top-down view of inlier blobs.

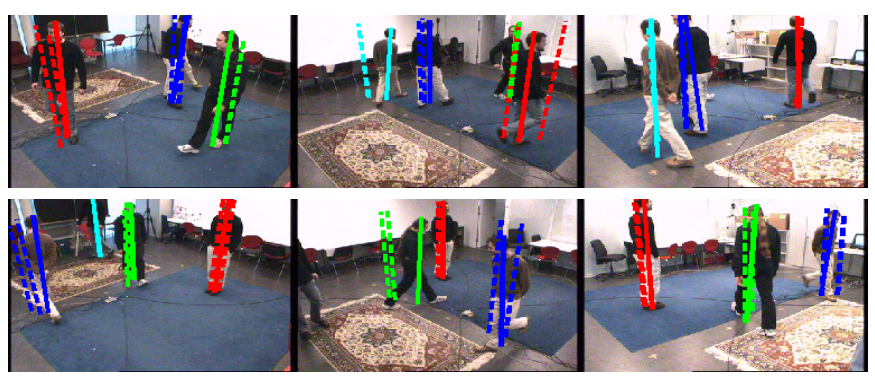

(a)
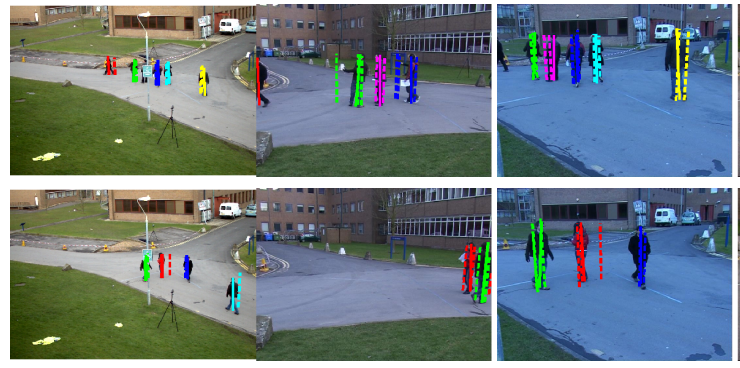

(c)
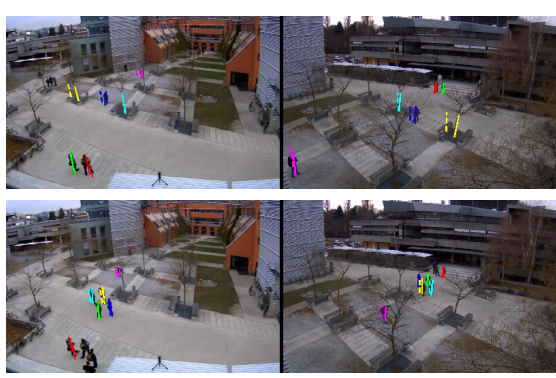

(b)

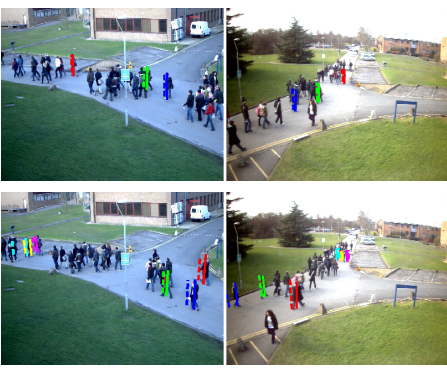

(d)

Figure 7. Re-projection evaluation. Manually labeled corresponding pedestrians in different views are plotted in straight-lines with the same color. The cross-view re-projections based on calibration are plotted in dashed lines. 


\section{Summary}

We propose a novel framework for unsupervised surveillance camera network calibration. We take noisy foreground (pedestrian) blobs captured directly by the cameras without any cross-time or cross-view correspondence information. We first apply robust self-calibration to calibrate each camera w.r.t. the IWCS to reduce the DoF of the projective transformation to be estimated later, meanwhile pruning a large proportion of outlier blob observations. We then sequentially align all the IWCSs to a shared gWCS, during which we introduce truncated least-squares as a robust error metric to iteratively determine inlier correspondences, while applying a series of partial DLTs to solve for a projective transformation. We demonstrate the robustness of our algorithm against different camera settings, foreground qualities, outlier ratios and crowd densities via extensive experiments on synthesized image sequences as well as on publicly available real datasets.

\section{Appendix: Groundplane Registration using Partial DLT}

Given fixed ground-plane rotation $\alpha$ and local calibration matrix $P_{L}$, we linearize the equation sets by introducing variable $z$ to resolve the ambiguity:

$$
\left[\begin{array}{c}
x \cdot z \\
y \cdot z \\
z
\end{array}\right]=P_{L} \cdot P_{G} \cdot\left[\begin{array}{c}
X \\
Y \\
Z \\
1
\end{array}\right]
$$

with

$$
\begin{aligned}
& P_{L}=\left[\begin{array}{llll}
p_{11} & p_{12} & p_{13} & p_{14} \\
p_{21} & p_{22} & p_{23} & p_{24} \\
p_{31} & p_{32} & p_{33} & p_{34}
\end{array}\right] \\
& P_{G}=\left[\begin{array}{rrrr}
\cos \alpha \cdot s, & -\sin \alpha \cdot s, & & t_{x} \\
\sin \alpha \cdot s, & \cos \alpha \cdot s, & & t_{y} \\
& & s & \\
& & & 1
\end{array}\right]
\end{aligned}
$$

Via Gaussian elimination on the auxiliary variable $z$, we can reorganize Eqn. 18 to obtain 2 sets of constrains for each $2 D-3 D$ point pair correspondence, so that the calibration parameters $\left[s, t_{x}, t_{y}\right]$ can be linearly solved:

$$
\left[\begin{array}{lll}
m_{11} & m_{12} & m_{13} \\
m_{21} & m_{22} & m_{23}
\end{array}\right] \cdot\left[\begin{array}{c}
s \\
t_{x} \\
t_{y}
\end{array}\right]=\left[\begin{array}{l}
u_{1} \\
u_{2}
\end{array}\right]
$$

where

$$
\begin{aligned}
m_{11}= & \left(p_{11} X+p_{12} Y-p_{31} x X-p_{32} x Y\right) \cos \alpha+\left(p_{12} X\right. \\
& \left.-p_{11} Y-p_{32} x X+p_{31} x Y\right) \sin \alpha+\left(p_{13}-p_{33} x\right) Z \\
m_{21}= & \left(p_{21} X+p_{22} Y-p_{31} y X-p_{32} y Y\right) \cos \alpha+\left(p_{22} X\right. \\
& \left.-p_{21} Y-p_{32} y X+p_{31} y Y\right) \sin \alpha+\left(p_{23}-p_{33} y\right) Z,
\end{aligned}
$$

and

$$
\begin{aligned}
m_{12} & =p_{11}-p_{31} x, & m_{22} & =p_{21}-p_{31} y, \\
m_{13} & =p_{12}-p_{32} x, & m_{23} & =p_{22}-p_{32} y \\
u_{1} & =-p_{14}+p_{34} x, & u_{2} & =-p_{24}+p_{34} y
\end{aligned}
$$

\section{References}

[1] J. Berclaz, F. Fleuret, E. Turetken, and P. Fua. Multiple Object Tracking using K-Shortest Paths Optimization. PAMI, 2011. 1, 5

[2] M. Black and P. Anandan. The robust estimation of multiple motions: Parametric and piecewise-smooth flow fields. CVIU, 63(1):75-104, 1996. 4

[3] T. Chen, A. Bimbo, F. Pernici, and G. Serra. Accurate selfcalibration of two cameras by observations of a moving person on a ground plane. In Proc. AVSS, 2007. 1

[4] W. Ge and R. T. Collins. Crowd detection with a multiview sampler. In Proc. ECCV, 2010. 1

[5] R. I. Hartley and A. Zisserman. Multiple View Geometry in Computer Vision. Cambridge University Press, ISBN: 0521623049, 2000. 3

[6] M. Hodlmoser and M. Kampel. Multiple camera selfcalibration and $3 \mathrm{D}$ reconstruction using pedestrians. In Proc. ISVC, 2010. 1

[7] I. N. Junejo and H. Foroosh. Trajectory rectification and path modeling for video surveillance. In Proc. ICCV, 2007. 1

[8] N. Krahnstoever and P. R. Mendonca. Bayesian autocalibration for surveillance. In Proc. ICCV, pages 1858-1865, 2005. 1,4

[9] D. Liebowitz. Camera Calibration and Reconstruction of Geometry from Images. PhD thesis, University of Oxford, 2001. 1

[10] D. Liebowitz and A. Zisserman. Combining scene and autocalibration constraints. In Proc. EuroGraphics, pages 293300, 1999. 2

[11] J. Liu, R. T. Collins, and Y. Liu. Surveillance camera autocalibration based on pedestrian height distributions. In Proc. BMVC, 2011. 1, 2, 3, 4, 5, 6, 8

[12] F. Lv, T. Zhao, and R. Nevatia. Camera calibration from video of a walking human. PAMI, 28(9):1513-1518, 2006. 1

[13] B. Micusik and T. Pajdla. Simultaneous surveillance camera calibration and foot-head homology estimation from human detections. In Proc. CVPR, 2010. 1

[14] H. Possegger, R. Matthias, S. Sabine, M. Thomas, K. Manfred, R. P. M., and B. Horst. Unsupervised calibration of camera networks and virtual PTZ cameras. In Computer Vision Winter Workshop, 2012. 1, 5

[15] D. Rother and K. A. Patwardhan. What can casual walkers tell us about the 3D scene. In Proc. ICCV, 2007. 1

[16] B. Triggs, P. McLauchlan, R. Hartley, and A. Fitzgibbon. Bundle adjustment-a modern synthesis. Vision Algorithms: Theory and Practice, 2000. 2 